\title{
Ações Disciplinares em Ginecologia e Obstetrícia na Região Norte de Portugal nos Anos 2008 a 2012
}

\author{
Disciplinary Actions in Gynecology and Obstetrics in the North of Portugal \\ from Year 2008 to 2012
}

Francisco SILVA 1 , Manuel RODRIGUES e RODRIGUES ${ }^{2}$, João BERNARDES ${ }^{2}$

Acta Med Port 2015 Mar-Apr;28(2):194-203

\section{RESUMO}

Introdução: As ações disciplinares podem ter um impacto significativo na vida dos médicos e dos doentes. O objetivo deste trabalho foi a avaliação das ações disciplinares em Ginecologia e Obstetrícia ocorridas na região norte de Portugal nos anos 2008 a 2012.

Material e Métodos: Estudo descritivo retrospetivo baseado nos dados anonimizados disponíveis nos relatórios anuais de atividades do Conselho Disciplinar da Seção Regional Norte da Ordem dos Médicos dos anos 2008 a 2012. Calculámos a proporção de ações disciplinares em Ginecologia e Obstetrícia sobre o número total de especialistas inscritos. Avaliámos também os tipos de queixoso, de arguido, de instituição, de queixa e de decisão envolvidos. Para comparação de proporções utilizámos intervalos de confiança de $95 \%$. Resultados: De 2008 a 2012, registou-se um total de 1040 ações disciplinares na região norte de Portugal. A Ginecologia e Obstetrícia foi a quarta especialidade mais afetada, com 54 queixas, 43 das quais relacionadas com alegada negligência médica e a mais afetada, se considerarmos apenas este tipo de queixa. Os queixosos e os arguidos mais frequentes foram, respetivamente, doentes em nome próprio e profissionais do sexo feminino, com idade compreendida entre os 41 e 60 anos. Das 54 ações disciplinares registadas, 52 foram concluídas sem penalização e duas aguardam decisão.

Discussão: Os resultados estão genericamente de acordo com os de outros autores.

Conclusões: A Ginecologia e Obstetrícia foi a quarta especialidade com maior risco de ação disciplinar na região norte de Portugal nos anos 2008 a 2012 e a primeira se considerarmos só as queixas de alegada negligência médica. Todas as ações disciplinares foram arquivadas, sem penalização dos médicos visados, com exceção de duas que se encontram ainda em avaliação.

Palavras-chave: Ginecologia; Negligência; Obstetrícia; Portugal; Responsabilidade Legal.

\section{ABSTRACT}

Introduction: Disciplinary actions may have a significant impact in medical doctors' and patients' lives. The objective of this study was the assessment of the disciplinary actions in Obstetrics and Gynecology that occurred in the north of Portugal in years 2008 to 2012.

Material and Methods: Retrospective descriptive study based on the anonymized data contained in the annual activity reports of Conselho Disciplinar da Seção Regional Norte da Ordem dos Médicos from 2008 to 2012. We calculated the proportion of disciplinary actions in Obstetrics and Gynaecology over the total number of registered specialists in that speciality. We also analysed the type of complainers, accused, institutions, complaints and decisions. For statistical inference proportions with $95 \%$ confidence intervals were estimated.

Results: From years 2008 to 2012, we registered 1040 complaints in all medical specialities in the north of Portugal. Obstetrics and Gynecology was the forth most affected specialty, with a total of 54 complaints. Forty-three complaints were related with medical malpractice and if we only consider this type of complaint Obstetrics and Gynecology was the most affected specialty. The most frequent complainers and accused were, respectively, patients themselves and female physicians, with 41 to 60 years of age. Fifty-two complaints were archived without punishment while two still await conclusion.

Discussion: The overall results of this study are in agreement with those reported by other authors.

Conclusions: Obstetrics and Gynecology was the forth speciality with highest risk for any disciplinary action in the north of Portugal in years 2008 to 2012 and the first one in relation with alleged negligence. All presented and already concluded complaints were archived without penalty, except two that are still under evaluation.

Keywords: Gynecology; Liability, Legal; Malpractice; Obstetrics; Portugal; Professional Misconduct.

\section{INTRODUÇÃO}

A profissão médica associa-se, desde tempos imemoriais, a uma forte regulamentação e escrutínio social, plasmados em inúmeros códigos, declarações e regulamentos, de diversas naturezas - ética, jurídica ou deontológica. ${ }^{1}$ Esta realidade é cada vez mais uma parte integrante do exercício profissional, condicionando-o profundamente. Calcula-se que, em média, os médicos possam estar envolvidos em ações disciplinares ou legais durante $11 \%$ dos 40 anos de vida média profissional. ${ }^{2}$ Trata-se, no entanto, de um assunto difícil de estudar, com pouca informação disponível na literatura médica. É grande a diversidade e com- plexidade dos processos, com os médicos a poderem ser alvo de ações movidas por entidades patronais, profissionais e judiciais. ${ }^{1} \dot{E}$, por isso, difícil obter um retrato completo dos resultados do escrutínio exercido sobre os médicos.

Alguns artigos e relatórios institucionais abordam os problemas no âmbito das ações movidas pelas entidades patronais, de natureza essencialmente administrativa, laboral e de qualidade de desempenho. ${ }^{3,4}$ Outros trabalhos interessam-se pelas ações movidas pelas associações profissionais, investidas de legitimidade deontológica e de poder disciplinar, como as ordens e os colégios de

1. Mestrado Integrado em Medicina. Faculdade de Medicina. Universidade do Porto. Porto. Portugal.

2. Conselho Disciplinar. Seção Regional Norte. Ordem dos Médicos. Porto. Portugal.

Recebido: 02 de Setembro de 2014 - Aceite: 04 de Fevereiro de 2015 | Copyright @ Ordem dos Médicos 2015 
médicos. ${ }^{5-7}$ Outros trabalhos, ainda, abordam as ações jurídicas, movidas pelos órgãos judiciais, Ministério Público e tribunais administrativos, penais ou civis. ${ }^{8} \mathrm{Em}$ todos os casos, é sempre difícil obter informações, por não existirem, em Portugal, bases de dados organizadas disponibilizadas ao público. Assim, os interessados em estudar o assunto têm de procurar investigar junto das entidades patronais, das associações profissionais e dos órgãos judiciais que tratam as diferentes dimensões da responsabilidade, nomeadamente o Serviço Nacional da Saúde/Ministério da Saúde, a Ordem dos Médicos (OM) e o Conselho Médico-Legal/Ministério da Justiça. As diferentes instâncias têm, dentro do seu âmbito de intervenção, mecanismos diferentes com legislação e penalizações próprias para tratar matérias do foro disciplinar. São exemplo disso, o Estatuto Disciplinar dos Trabalhadores Que Exercem Funções Públicas e o Estatuto da Ordem dos Médicos que, aplicando-se a um mesmo médico, têm âmbitos de ação diferentes, um na responsabilidade administrativa e outro na responsabilidade profissional e deontológica. ${ }^{1,9,10}$

Seja qual for o cenário de escrutínio do exercício da profissão médica, a especialidade de Ginecologia e Obstetrícia afigura-se como uma das mais críticas, ${ }^{8,11-15}$ aparecendo como uma das que apresenta maior proporção de ações disciplinares e judiciais, bem como de penas e de pedidos indemnizatórios mais elevados. ${ }^{13-15}$ Em Portugal conhecemos as queixas que deram entrada no Conselho Médico-Legal do Ministério da Justiça, no âmbito da Ginecologia e Obstetrícia, ${ }^{8}$ mas não conhecemos nenhuma publicação que analise as ações de ordem disciplinar da esfera de competência da Ordem dos Médicos. Nesse sentido, o objetivo deste trabalho foi a avaliação das ações disciplinares em Ginecologia e Obstetrícia que deram entrada no Conselho Disciplinar (CD) da Seção Regional Norte (SRN) da OM, de Portugal, nos anos 2008 a 2012.
Os Conselhos Disciplinares (Nacional, Norte, Centro e Sul e Ilhas) são os órgãos estatutariamente competentes para a instauração de processos disciplinares na OM. É evidente a importância destes órgãos no cumprimento das funções da OM, em particular no zelar pelo cumprimento do Código Deontológico e do Estatuto Disciplinar dos Médicos. ${ }^{16,17}$ É função destes conselhos lidar com a responsabilidade ético-profissional, cabendo as demais responsabilidades (civil e criminal) às instâncias competentes no quadro legal português - o Ministério Público e os tribunais, à semelhança do que acontece noutros países. ${ }^{18-22}$

\section{MATERIAL E MÉTODOS}

Estudo descritivo retrospetivo, aprovado pelo CD da SRN da OM, baseado nos dados anonimizados disponíveis nos relatórios anuais de atividades e na consulta manual dos processos da especialidade de Ginecologia e Obstetrícia envolvidos, realizada por um dos membros do CD. Construímos uma base de dados a partir dos relatórios referidos, tendo sido analisados todos os processos que foram instaurados desde 1 de janeiro de 2008 a 31 de dezembro de 2012, independentemente de estarem ou não concluídos.

O CD da SRN da OM é constituído por cinco médicos, assessorados por dois juristas e é responsável pela análise e decisão das queixas apresentadas contra médicos inscritos na SRN da OM. Desde o momento de apresentação de uma queixa, esta segue a tramitação estipulada, à semelhança do que acontece noutras associações profissionais que tutelam a prática médica. ${ }^{19} \mathrm{O}$ procedimento inclui, sequencialmente, a identificação dos intervenientes e a análise sumária da queixa, seguida de arquivamento imediato ou, no caso de abertura de um processo disciplinar, da distribuição por um relator responsável por apresentar o caso ao CD, que determina o pedido, ou não, de

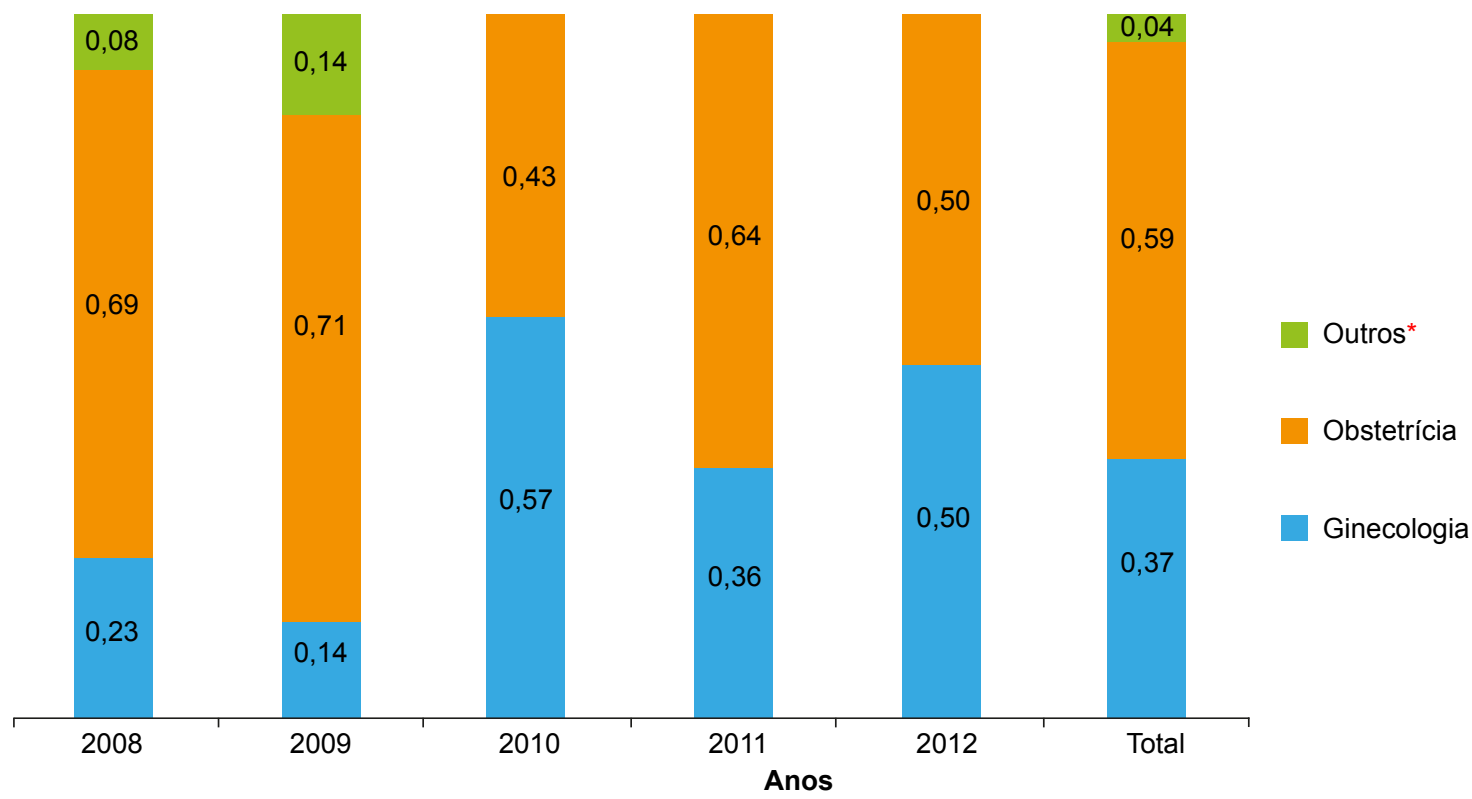

Figura 1 - Distribuição das ações disciplinares em Ginecologia e Obstetrícia, na região norte de Portugal, nos anos 2008 a 2012 , por área da especialidade. *casos não incluídos nas áreas anteriores. 
Tabela 1 - Proporções médias gerais de acções disciplinares, por ano e por especialidade, no norte de Portugal, no quinquénio 2008-2012, com

\begin{tabular}{|c|c|c|c|c|c|c|c|c|c|}
\hline \multirow[t]{2}{*}{ Especialidade } & \multicolumn{3}{|c|}{2008} & \multicolumn{3}{|c|}{2009} & \multicolumn{3}{|c|}{2010} \\
\hline & $\mathbf{n}$ & Esp & Prop & $\mathbf{n}$ & Esp & Prop & $\mathbf{n}$ & Esp & Prop \\
\hline Psiquiatria & 9 & 302 & 0,03 & 6 & 309 & 0,02 & 4 & 318 & 0,01 \\
\hline Ortopedia & 7 & 310 & 0,02 & 8 & 316 & 0,03 & 10 & 330 & 0,03 \\
\hline Radiologia & 4 & 220 & 0,02 & 10 & 226 & 0,04 & 7 & 235 & 0,03 \\
\hline Ginecologia e Obstetrícia & 13 & 445 & 0,03 & 7 & 452 & 0,02 & 14 & 461 & 0,03 \\
\hline Cirurgia Plástica, Reconstrutiva e Estética & 2 & 59 & 0,03 & 1 & 61 & 0,02 & 3 & 60 & 0,05 \\
\hline Oftalmologia & 6 & 219 & 0,03 & 0 & 222 & 0,00 & 5 & 230 & 0,02 \\
\hline Gastrenterologia & 1 & 116 & 0,01 & 1 & 118 & 0,01 & 4 & 121 & 0,03 \\
\hline Medicina Geral e Familiar & 27 & 1742 & 0,02 & 28 & 1786 & 0,02 & 21 & 1836 & 0,01 \\
\hline Cirurgia Geral & 13 & 462 & 0,03 & 4 & 474 & 0,01 & 5 & 479 & 0,01 \\
\hline Medicina do Trabalho & 1 & 278 & 0,00 & 7 & 282 & 0,02 & 6 & 290 & 0,02 \\
\hline Otorrinolaringologia & 0 & 172 & 0,00 & 6 & 177 & 0,03 & 1 & 183 & 0,01 \\
\hline Reumatologia & 1 & 25 & 0,04 & 0 & 27 & 0,00 & 0 & 28 & 0,00 \\
\hline Urologia & 3 & 111 & 0,03 & 0 & 113 & 0,00 & 0 & 117 & 0,00 \\
\hline Angiologia e Cirurgia Vascular & 3 & 62 & 0,05 & 1 & 61 & 0,02 & 0 & 62 & 0,00 \\
\hline Neurocirurgia & 0 & 51 & 0,00 & 0 & 51 & 0,00 & 1 & 51 & 0,02 \\
\hline Cardiologia & 4 & 212 & 0,02 & 1 & 215 & 0,00 & 3 & 219 & 0,01 \\
\hline Neurologia & 2 & 120 & 0,02 & 0 & 125 & 0,00 & 3 & 128 & 0,02 \\
\hline Estomatologia & 4 & 204 & 0,02 & 1 & 199 & 0,01 & 2 & 198 & 0,01 \\
\hline Medicina Física e Reabilitação & 1 & 178 & 0,01 & 4 & 184 & 0,02 & 1 & 192 & 0,01 \\
\hline Oncologia Médica & 1 & 84 & 0,01 & 0 & 87 & 0,00 & 2 & 85 & 0,02 \\
\hline Genética Médica & 1 & 23 & 0,04 & 0 & 24 & 0,00 & 0 & 24 & 0,00 \\
\hline Psiquiatria da Infância e da Adolescência & 0 & 48 & 0,00 & 1 & 49 & 0,02 & 1 & 49 & 0,02 \\
\hline Endocrinologia & 1 & 60 & 0,02 & 0 & 60 & 0,00 & 1 & 62 & 0,02 \\
\hline Saúde Pública & 1 & 136 & 0,01 & 2 & 138 & 0,01 & 0 & 138 & 0,00 \\
\hline Radioterapia & 1 & 33 & 0,03 & 0 & 34 & 0,00 & 0 & 34 & 0,00 \\
\hline Imuno-hemoterapia & 0 & 75 & 0,00 & 0 & 74 & 0,00 & 2 & 74 & 0,03 \\
\hline Nefrologia & 0 & 71 & 0,00 & 1 & 76 & 0,01 & 1 & 78 & 0,01 \\
\hline Não Especialistas/Clínica Geral & 38 & 5195 & 0,01 & 23 & 5504 & 0,00 & 22 & 5967 & 0,00 \\
\hline Dermato-Venereologia & 1 & 83 & 0,01 & 0 & 85 & 0,00 & 0 & 85 & 0,00 \\
\hline Medicina Interna & 1 & 520 & 0,00 & 2 & 539 & 0,00 & 2 & 551 & 0,00 \\
\hline Pneumologia & 1 & 139 & 0,01 & 2 & 143 & 0,01 & & 144 & 0,00 \\
\hline Pediatria & 3 & 488 & 0,01 & 3 & 515 & 0,01 & 2 & 528 & 0,00 \\
\hline Imuno-alergologia & 0 & 55 & 0,00 & 1 & 57 & 0,02 & 0 & 60 & 0,00 \\
\hline Anestesiologia & 1 & 497 & 0,00 & 1 & 526 & 0,00 & 1 & 548 & 0,00 \\
\hline Total & 206 & 14143 & 0,01 & 179 & 14705 & 0,01 & 154 & 15416 & 0,01 \\
\hline
\end{tabular}

Esp: especialistas; Prop: proporção; Prop MAC: proporção média anual cumulativa

pareceres (nomeadamente aos Colégios de Especialidade, nos casos de alegada má-prática), a audição, ou não, de testemunhas e a proferição de um despacho de arquivamento ou de acusação. Neste último caso, segue-se a audiência e defesa (por escrito) do arguido e, finalmente, a emissão de um despacho de arquivamento ou de condenação. ${ }^{17}$

Avaliámos a proporção de ações disciplinares em Ginecologia e Obstetrícia sobre o número total de especialistas inscritos, comparando-a com as de outras especialidades (Tabela 1). Foram também analisados os tipos de queixoso, de arguido, de queixa e de decisão envolvidos (Tabelas $2 \mathrm{e}$ 3).

Para a análise da proporção de queixas apresentadas por especialidade, solicitámos à SRN da OM o número de médicos inscritos em cada especialidade, nos anos de 2008 a 2012.

Relativamente às queixas apresentadas, optámos por uma análise que permitisse a avaliação demográfica de cada profissional envolvido, uma vez que uma queixa poderia incluir vários profissionais, com desfechos eventualmente diferentes para cada um dos visados. Cada processo foi então considerado como sendo a instauração de um procedimento disciplinar a um médico.

Caracterizámos cada procedimento disciplinar com base em diferentes variáveis, com vista à respetiva análise estatística. Quanto ao motivo, classificámos as queixas nas categorias adotadas nos relatórios anuais do CD da SRN 
os respetivos intervalos de confiança de 95\% (IC 95\%) ordenadas por ordem decrescente

\begin{tabular}{|c|c|c|c|c|c|c|c|c|c|}
\hline \multicolumn{3}{|c|}{2011} & \multicolumn{3}{|c|}{2012} & \multicolumn{4}{|c|}{ Cumulativo 2008-12 } \\
\hline $\mathbf{n}$ & Esp & Prop & $\mathbf{n}$ & Esp & Prop & $\mathbf{n}$ & Esp & Prop MAC & IC95\% \\
\hline 22 & 329 & 0,07 & 23 & 341 & 0,07 & 64 & 1599 & 0,040 & $(0,031-0,051)$ \\
\hline 19 & 346 & 0,05 & 18 & 357 & 0,05 & 62 & 1659 & 0,037 & $(0,029-0,048)$ \\
\hline 3 & 250 & 0,01 & 6 & 261 & 0,02 & 30 & 1192 & 0,025 & $(0,018-0,036)$ \\
\hline 14 & 472 & 0,03 & 6 & 485 & 0,01 & 54 & 2315 & 0,023 & $(0,018--0,030)$ \\
\hline 0 & 61 & 0,00 & 1 & 63 & 0,02 & 7 & 304 & 0,023 & $(0,011 .-0,047)$ \\
\hline 5 & 237 & 0,02 & 9 & 251 & 0,04 & 25 & 1159 & 0,022 & $(0,015-0,032)$ \\
\hline 2 & 128 & 0,02 & 5 & 135 & 0,04 & 13 & 618 & 0,021 & $(0,012-0,036)$ \\
\hline 69 & 1892 & 0,04 & 29 & 1977 & 0,01 & 174 & 9233 & 0,019 & $(0,016-0,022)$ \\
\hline 8 & 489 & 0,02 & 12 & 510 & 0,02 & 42 & 2414 & 0,017 & $(0,013-.0,023)$ \\
\hline 4 & 298 & 0,01 & 6 & 298 & 0,02 & 24 & 1446 & 0,017 & $(0,011-0,025)$ \\
\hline 2 & 191 & 0,01 & 4 & 197 & 0,02 & 13 & 920 & 0,014 & $(0,008-0,024)$ \\
\hline 0 & 31 & 0,00 & 1 & 33 & 0,03 & 2 & 144 & 0,014 & $(0,004-0,049)$ \\
\hline 2 & 123 & 0,02 & 3 & 127 & 0,02 & 8 & 591 & 0,014 & $(0,007-0,026)$ \\
\hline 0 & 64 & 0,00 & 0 & 68 & 0,00 & 4 & 317 & 0,013 & $(0,005-0,032)$ \\
\hline 1 & 50 & 0,02 & 1 & 51 & 0,02 & 3 & 254 & 0,012 & $(0,004-0,034)$ \\
\hline 1 & 223 & 0,00 & 3 & 239 & 0,01 & 12 & 1108 & 0,011 & $(0,006-0,019)$ \\
\hline 1 & 136 & 0,01 & 1 & 144 & 0,01 & 7 & 653 & 0,011 & $(0,005-0,022)$ \\
\hline 1 & 192 & 0,01 & 2 & 188 & 0,01 & 10 & 981 & 0,010 & $(0,006-0,019)$ \\
\hline 1 & 194 & 0,01 & 2 & 202 & 0,01 & 9 & 950 & 0,009 & $(0,005-0,018)$ \\
\hline 0 & 88 & 0,00 & 1 & 101 & 0,01 & 4 & 445 & 0,009 & $(0,004-0,023)$ \\
\hline 0 & 26 & 0,00 & 0 & 27 & 0,00 & 1 & 124 & 0,008 & $(0,001-0,044)$ \\
\hline 0 & 55 & 0,00 & 0 & 58 & 0,00 & 2 & 259 & 0,008 & $(0,002-0,028)$ \\
\hline 0 & 69 & 0,00 & 0 & 75 & 0,00 & 2 & 326 & 0,006 & $(0,002-0,022)$ \\
\hline 0 & 140 & 0,00 & 1 & 144 & 0,01 & 4 & 696 & 0,006 & $(0,002-0,015)$ \\
\hline 0 & 36 & 0,00 & 0 & 40 & 0,00 & 1 & 177 & 0,006 & $(0,001-0,031)$ \\
\hline 0 & 74 & 0,00 & 0 & 78 & 0,00 & 2 & 375 & 0,005 & $(0,001-0,019)$ \\
\hline 0 & 83 & 0,00 & 0 & 86 & 0,00 & 2 & 394 & 0,005 & $(0,001-0,018)$ \\
\hline 14 & 6350 & 0,00 & 36 & 6234 & 0,01 & 133 & 29250 & 0,005 & $(0,004-0,005)$ \\
\hline 0 & 90 & 0,00 & 1 & 97 & 0,01 & 2 & 440 & 0,005 & $(0,001-0,016)$ \\
\hline 2 & 578 & 0,00 & 5 & 629 & 0,01 & 12 & 2817 & 0,004 & $(0,002-0,007)$ \\
\hline 0 & 145 & 0,00 & 0 & 156 & 0,00 & 3 & 727 & 0,004 & $(0,001-0,012)$ \\
\hline 0 & 545 & 0,00 & 2 & 604 & 0,00 & 10 & 2680 & 0,004 & $(0,002-0,007)$ \\
\hline 0 & 63 & 0,00 & 0 & 63 & 0,00 & 1 & 298 & 0,003 & $(0,001-0,019)$ \\
\hline 1 & 574 & 0,00 & 1 & 600 & 0,00 & 5 & 2745 & 0,002 & $(0,001 .-0,004)$ \\
\hline 230 & 16151 & 0,01 & 271 & 16520 & 0,02 & 1040 & 76935 & 0,014 & $(0,013-0,014)$ \\
\hline
\end{tabular}

da OM, que incluíam negligência médica, conflito médico/ doente e conflito médico/médico, entre outras. A classificação da penalização seguiu o Estatuto Disciplinar dos Médicos quanto ao tipo de sanções a aplicar: advertência, censura, suspensão (até 5 anos) ou expulsão. ${ }^{17}$ Os queixosos foram classificados em quatro categorias, consoante a proveniência das queixas: médico, doente, instituições ou outros (incluindo nesta última, advogados, na qualidade de mandatários do respetivo cliente). Os médicos visados foram avaliados quanto ao género e grupo etário e descrevemos o local de ocorrência dos factos: hospitais públicos, hospitais privados ou clínicas médicas, entre outros (Tabela 2).

Utilizámos, de uma forma geral, as designações pro- cessuais adotadas pelo $C D$, tais como "arguido", "negligência" ou "acusação", para nos mantermos fieis à linguagem habitualmente utilizada.

Analisámos os dados recolhidos com o Microsoft Excel for Mac $2011^{\circledR}$. Para inferência estatística avaliámos proporções com intervalos de confiança de 95\%.

\section{RESULTADOS}

Nos cinco anos analisados deram entrada, no CD da SRN da OM, 866 queixas que deram origem a 1040 processos disciplinares, tendo 54 processos correspondido a queixas contra médicos no âmbito da Ginecologia e Obstetrícia. A Obstetrícia foi a área que registou mais queixas: $59,3 \%$ versus $37,0 \%$ na Ginecologia; os restantes 3,7\% es- 
tavam relacionados com comportamentos profissionais incorretos não relacionados diretamente com a prática clínica (Fig. 1). Considerando o número de profissionais inscritos na SRN da OM, a Ginecologia e Obstetrícia foi a quarta especialidade mais afetada, a seguir à Psiquiatria, Ortopedia e Radiologia (Tabela 1). Contudo, se só considerarmos as queixas de alegada negligência médica, a Ginecologia e
Obstetrícia foi a especialidade mais afetada (Tabela 4).

Das cinquenta e quatro queixas, 43 estavam relacionadas com alegada negligência médica, oito com conflitos médico/doente, uma com conflito médico/médico, uma com conluio médico/comércio e uma com erro de diagnóstico (Tabela 3). Os queixosos mais frequentes (Tabela 2) foram doentes em nome próprio $(66,7 \%)$, seguidos de instituições

Tabela 2 - Tipo de queixosos, de arguidos e de locais implicados nas ações disciplinares em Ginecologia e Obstetrícia, na região norte do país, nos anos 2008-2012

\begin{tabular}{|c|c|c|c|c|c|c|}
\hline & & & Anos & & & \\
\hline & 2008 & 2009 & 2010 & 2011 & 2012 & Total \\
\hline Queixoso & & & & & & \\
\hline Tipo & & & & & & \\
\hline Doente & 3 & 6 & 8 & 13 & 6 & 36 \\
\hline Instituição & 6 & 1 & 5 & - & - & 12 \\
\hline Médico & 1 & - & - & - & - & 1 \\
\hline Outros & 3 & - & 1 & 1 & . & 5 \\
\hline Total & 13 & 7 & 14 & 14 & 6 & 54 \\
\hline Género & & & & & & \\
\hline Feminino & 10 & 1 & 14 & 9 & 3 & 37 \\
\hline Masculino & 3 & 5 & - & 5 & 3 & 16 \\
\hline Não aplicável & - & $1^{*}$ & - & - & - & 1 \\
\hline Total & 13 & 7 & 14 & 14 & 6 & 54 \\
\hline Acusado & & & & & & \\
\hline Género & & & & & & \\
\hline Feminino & 11 & 6 & 6 & 8 & 2 & 33 \\
\hline Masculino & 2 & 1 & 8 & 6 & 4 & 21 \\
\hline Total & 13 & 7 & 14 & 14 & 6 & 54 \\
\hline Idade & & & & & & \\
\hline $26-30$ & - & 2 & - & - & - & 2 \\
\hline $31-35$ & 2 & - & 3 & - & 1 & 6 \\
\hline $36-40$ & - & 1 & - & 1 & - & 2 \\
\hline $41-45$ & 3 & 1 & 1 & 1 & 1 & 7 \\
\hline $46-50$ & 2 & 1 & 2 & 4 & - & 9 \\
\hline $51-55$ & 4 & 1 & 3 & 2 & 3 & 13 \\
\hline $56-60$ & 1 & 1 & 2 & 5 & 1 & 10 \\
\hline $61-65$ & 1 & - & 3 & - & - & 4 \\
\hline $66-70$ & - & - & - & 1 & - & 1 \\
\hline Total & 13 & 7 & 14 & 14 & 6 & 54 \\
\hline Local & & & & & & \\
\hline Hospitais Públicos & 10 & 4 & 12 & 5 & 3 & 34 \\
\hline Clínicas Médicas & 2 & 2 & - & 5 & 1 & 10 \\
\hline Casas de Saúde & - & - & 2 & 2 & - & 4 \\
\hline Hospitais Particulares & 1 & - & - & 1 & 2 & 4 \\
\hline Consultórios Médicos & - & 1 & - & 1 & - & 2 \\
\hline Total & 13 & 7 & 14 & 14 & 6 & 54 \\
\hline
\end{tabular}

*processo instaurado por conluio médico-comércio. 
$(22,2 \%)$ - que incluíram Administrações de Centros Hospitalares, a Entidade Reguladora da Saúde, o Ministério Público ou o Ministério da Saúde - e de advogados na qualidade de mandatários do doente $(9,3 \%)$.

Todos os arguidos, à exceção de um, eram de nacionalidade portuguesa. O perfil demográfico do visado (Tabela 2) caracterizou-se por serem predominantemente do sexo feminino $(61,1 \%)$, com 41 e 60 anos $(72,2 \%)$, tendo oito dos 54 processos sido instaurados a internos de formação específica.

Quanto ao local de ocorrência, 63\% associaram-se a hospitais públicos, $18,5 \%$ a clínicas médicas, $7,4 \%$ a hospitais particulares, $7,4 \%$ a casas de Saúde e 3,7\% a Consultórios médicos (Tabela 2).

As circunstâncias que motivaram as queixas em Obstetrícia (Fig. 2-A), relacionaram-se essencialmente com: Asfixia perinatal relacionada com distocia de ombros ou paralisia cerebral; erros de diagnóstico pré-natal ecográfico; complicações maternas de rutura uterina, laqueação das trompas durante cesariana sem consentimento escrito, fís- tula reto-vaginal ou necessidade de reintervenções; lesões traumáticas do recém-nascido secundárias à aplicação de fórceps; e abortamento tardio. Quanto à Ginecologia (Fig. 2-B), temos a considerar: Complicações cirúrgicas e pós-cirúrgicas relacionadas com intervenções de correção de incontinência urinária, conização, endometriose pélvica profunda, histerectomia, malformação uterina ou histeroscopia; situações de avaliação e/ou seguimento inapropriados e erros de diagnóstico. Os conflitos na relação médico/ doente (nomeadamente atrasos no atendimento de doentes ou comportamento médico alegadamente inapropriado) foram transversais a ambas as áreas.

Dos processos analisados, 52 foram arquivados, e dois (um por conflito médico/doente e outro por conluio médico/ comércio) continuam a aguardar decisão (Tabela 3).

\section{DISCUSSÃO}

Neste estudo descritivo retrospetivo, avaliámos as ações disciplinares em Ginecologia e Obstetrícia ocorridas na região norte de Portugal nos anos 2008 a 2012, a partir

Tabela 3 - Tipo de despachos de acusação por queixas disciplinares em Ginecologia e Obstetrícia, nos anos 2008-2012

\begin{tabular}{|c|c|c|c|c|c|c|}
\hline & 2008 & 2009 & 2010 & 2011 & 2012 & Total \\
\hline Conflito Médico(a)/Doente & - & 2 & 2 & 2 & 2 & 8 \\
\hline Arquivamento/ Sem Penalização & - & 1 & 2 & 2 & - & 5 \\
\hline Não concluído & - & 1 & - & - & 2 & 3 \\
\hline Conflito Médico/Médico & 1 & - & - & - & - & 1 \\
\hline Arquivamento/ Sem Penalização & 1 & - & - & - & - & 1 \\
\hline Conluio Médico/Comércio & - & 1 & - & - & - & 1 \\
\hline Não concluído & - & 1 & - & - & - & 1 \\
\hline Erro de Diagnóstico & - & - & - & 1 & - & 1 \\
\hline Arquivamento/ Sem Penalização & - & - & - & 1 & - & 1 \\
\hline Negligência Médica & 12 & 4 & 12 & 11 & 4 & 43 \\
\hline Arquivamento/ Sem Penalização & 12 & 4 & 12 & 11 & 4 & 43 \\
\hline Total & 13 & 7 & 14 & 14 & 6 & 54 \\
\hline
\end{tabular}
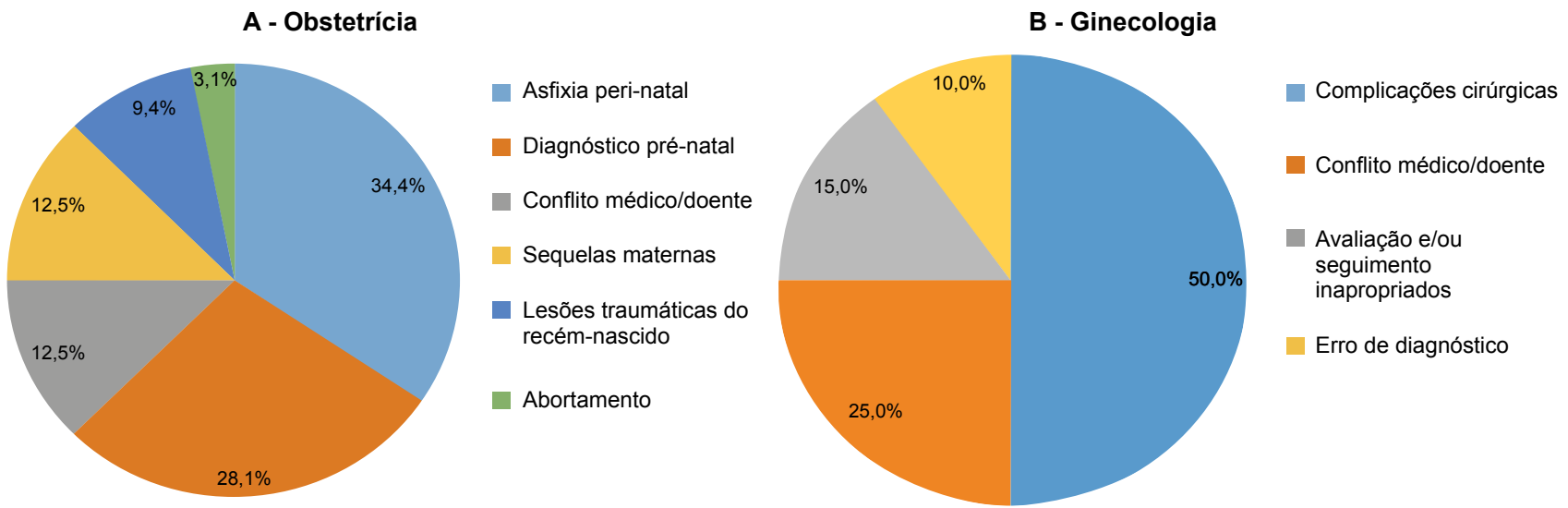

Figura 2 - Motivos das queixas de negligência médica em Obstetrícia (A) e Ginecologia (B), na região norte de Portugal, nos anos 2008 a 2012 
Tabela 4 - Proporções médias de acções disciplinares por alegada negligência, por ano e por especialidade, no norte de Portugal, no quinquénio

\begin{tabular}{|c|c|c|c|c|c|c|c|c|c|}
\hline \multirow[t]{2}{*}{ Especialidade } & \multicolumn{3}{|c|}{2008} & \multicolumn{3}{|c|}{2009} & \multicolumn{3}{|c|}{2010} \\
\hline & $\mathbf{n}$ & Esp & Prop & $\mathbf{n}$ & Esp & Prop & $\mathbf{n}$ & Esp & Prop \\
\hline Ginecologia e Obstetrícia & 12 & 445 & 0,03 & 4 & 452 & 0,01 & 12 & 461 & 0,03 \\
\hline Ortopedia & 3 & 310 & 0,01 & 6 & 316 & 0,02 & 4 & 330 & 0,01 \\
\hline Radiologia & 3 & 220 & 0,01 & 7 & 226 & 0,03 & 5 & 235 & 0,02 \\
\hline Cirurgia Plástica, Reconstrutiva e Estética & 1 & 59 & 0,02 & 1 & 61 & 0,02 & 2 & 60 & 0,03 \\
\hline Angiologia e Cirurgia Vascular & 3 & 62 & 0,05 & 1 & 61 & 0,02 & 0 & 62 & 0,00 \\
\hline Otorrinolaringologia & 0 & 172 & 0,00 & 6 & 177 & 0,03 & 1 & 183 & 0,01 \\
\hline Cirurgia Geral & 8 & 462 & 0,02 & 1 & 474 & 0,00 & 2 & 479 & 0,00 \\
\hline Oftalmologia & 4 & 219 & 0,02 & 0 & 222 & 0,00 & 4 & 230 & 0,02 \\
\hline Urologia & 3 & 111 & 0,03 & 0 & 113 & 0,00 & 0 & 117 & 0,00 \\
\hline Genética Médica & 1 & 23 & 0,04 & 0 & 24 & 0,00 & 0 & 24 & 0,00 \\
\hline Neurocirurgia & 0 & 51 & 0,00 & 0 & 51 & 0,00 & 1 & 51 & 0,02 \\
\hline Reumatologia & 1 & 25 & 0,04 & 0 & 27 & 0,00 & 0 & 28 & 0,00 \\
\hline Gastrenterologia & 0 & 116 & 0,00 & 0 & 118 & 0,00 & 1 & 121 & 0,01 \\
\hline Endocrinologia & 1 & 60 & 0,02 & 0 & 60 & 0,00 & 0 & 62 & 0,00 \\
\hline Neurologia & 1 & 120 & 0,01 & 0 & 125 & 0,00 & 0 & 128 & 0,00 \\
\hline Medicina Geral e Familiar & 5 & 1742 & 0,00 & 6 & 1786 & 0,00 & 6 & 1836 & 0,00 \\
\hline Imuno-hemoterapia & 0 & 75 & 0,00 & 0 & 74 & 0,00 & 1 & 74 & 0,01 \\
\hline Dermato-Venereologia & 1 & 83 & 0,01 & 0 & 85 & 0,00 & 0 & 85 & 0,00 \\
\hline Oncologia Médica & 1 & 84 & 0,01 & 0 & 87 & 0,00 & 0 & 85 & 0,00 \\
\hline Medicina Física e Reabilitação & 0 & 178 & 0,00 & 1 & 184 & 0,01 & 0 & 192 & 0,00 \\
\hline Estomatologia & 1 & 204 & 0,00 & 0 & 199 & 0,00 & 0 & 198 & 0,00 \\
\hline Não Especialistas/Clínica Geral & 8 & 5195 & 0,00 & 11 & 5504 & 0,00 & 14 & 5967 & 0,00 \\
\hline Cardiologia & 1 & 212 & 0,00 & 0 & 215 & 0,00 & 1 & 219 & 0,00 \\
\hline Medicina Interna & 0 & 520 & 0,00 & 0 & 539 & 0,00 & 2 & 551 & 0,00 \\
\hline Pediatria & 2 & 488 & 0,00 & 1 & 515 & 0,00 & 0 & 528 & 0,00 \\
\hline Anestesiologia & 1 & 497 & 0,00 & 0 & 526 & 0,00 & 0 & 548 & 0,00 \\
\hline Medicina do Trabalho & 0 & 278 & 0,00 & 0 & 282 & 0,00 & 1 & 290 & 0,00 \\
\hline Psiquiatria & 0 & 302 & 0,00 & 1 & 309 & 0,00 & 0 & 318 & 0,00 \\
\hline TOTAL & 80 & 14143 & 0,01 & 70 & 14705 & 0,00 & 67 & 15416 & 0,00 \\
\hline
\end{tabular}

de dados anonimizados disponíveis nos relatórios anuais de atividades do CD da SRN da OM e da consulta manual dos processos. Dessa forma, tivemos apenas acesso a um número limitado de dados, deixando algumas questões em aberto para estudos futuros. Para a classificação dos motivos, seguimos as categorias adoptadas nos relatórios anuais do CD. Seria interessante uma análise dos contornos específicos de cada processo, para distinguir em que circunstâncias se aplicam os conceitos de negligência médica, má prática, erro e efeitos adversos, entre outros. A classificação adoptada pelo $C D$ poderá ser diferente da de trabalhos apresentados por autores de outros países, pelo que a comparação deverá ser feita tendo em conta as dife- renças próprias de cada país, na perceção da responsabilidade dos médicos. ${ }^{20,23}$ Por exemplo, em relação às queixas classificadas como negligência, para alguns autores esta é equivalente a má prática, pelo não cumprimento da leges artis, enquanto que para outros a negligência implica uma componente de dolo, ${ }^{20,23}$ sendo que os contornos entre uma e outra situação não são sempre claros. ${ }^{24} \mathrm{O}$ desenvolvimento de bases de dados padronizadas, com a emissão de relatórios públicos anuais anonimizados e de sensibilização seria interessante, a par dos relatos de casos-tipo, publicados regularmente na Revista da Ordem dos Médicos, sabendo-se que tal poderá ter um impacto pedagógico positivo a nível da pré-graduação, da formação complementar 
2008-2012, com os respetivos intervalos de confiança de 95\% (IC 95\%), ordenadas por ordem decrescente

\begin{tabular}{|c|c|c|c|c|c|c|c|c|c|}
\hline \multicolumn{3}{|c|}{2011} & \multicolumn{3}{|c|}{2012} & \multicolumn{4}{|c|}{ Cumulativo 2008-12 } \\
\hline 11 & 472 & 0,02 & 4 & 485 & 0,01 & 43 & 2315 & 0,019 & $(0,014-0,025)$ \\
\hline 7 & 346 & 0,02 & 10 & 357 & 0,03 & 30 & 1659 & 0,018 & $(0,013-0,026)$ \\
\hline 2 & 250 & 0,01 & 4 & 261 & 0,02 & 21 & 1192 & 0,018 & $(0,012-0,027)$ \\
\hline 0 & 191 & 0,00 & 3 & 197 & 0,02 & 10 & 920 & 0,011 & $(0,006-0,020)$ \\
\hline 4 & 489 & 0,01 & 10 & 510 & 0,02 & 25 & 2414 & 0,010 & $(0,007-0,015)$ \\
\hline 0 & 237 & 0,00 & 4 & 251 & 0,02 & 12 & 1159 & 0,010 & $(0,006-0,018)$ \\
\hline 0 & 31 & 0,00 & 0 & 33 & 0,00 & 1 & 144 & 0,007 & $(0,001-0,038)$ \\
\hline 1 & 128 & 0,01 & 2 & 135 & 0,01 & 4 & 618 & 0,006 & $(0,003-0,017)$ \\
\hline 0 & 69 & 0,00 & 0 & 75 & 0,00 & 1 & 326 & 0,003 & $(0,001-0,017)$ \\
\hline 1 & 136 & 0,01 & 0 & 144 & 0,00 & 2 & 653 & 0,003 & $(0,001-0,011)$ \\
\hline 6 & 1892 & 0,00 & 5 & 1977 & 0,00 & 28 & 9233 & 0,003 & $(0,002-0,004)$ \\
\hline 0 & 74 & 0,00 & 0 & 78 & 0,00 & 1 & 375 & 0,003 & $(0,000-0,015)$ \\
\hline 0 & 90 & 0,00 & 0 & 97 & 0,00 & 1 & 440 & 0,002 & $(0,000-0,013)$ \\
\hline 0 & 88 & 0,00 & 0 & 101 & 0,00 & 1 & 445 & 0,002 & $(0,000-0,013)$ \\
\hline 0 & 545 & 0,00 & 1 & 604 & 0,00 & 4 & 2680 & 0,001 & $(0,001-0,004)$ \\
\hline 0 & 574 & 0,00 & 1 & 600 & 0,00 & 2 & 2745 & 0,001 & $(0,000-0,003)$ \\
\hline 0 & 298 & 0,00 & 0 & 298 & 0,00 & 1 & 1446 & 0,001 & $(0,000-0,004)$ \\
\hline 0 & 329 & 0,00 & 0 & 341 & 0,00 & 1 & 1599 & 0,001 & $(0,000-0,004)$ \\
\hline 61 & 16151 & 0,00 & 73 & 16520 & 0,00 & 351 & 76935 & 0,005 & $(0,004-0,005)$ \\
\hline
\end{tabular}

na especialidade e da formação profissional contínua. . $^{825,26}$

De 2008 a 2012, a proporção total de ações disciplinares foi sensivelmente constante (proporção $\approx 0,01$ ) (Tabela 1), tendo a Ginecologia e Obstetrícia sido a quarta especialidade mais afetada, com 54 queixas, predominantemente relacionadas com alegada negligência (Tabela 3 ). Estes dados estão de acordo com a literatura que coloca a Ginecologia e Obstetrícia como uma das especialidades mais propensa ao litígio médico-legal (Tabela 4). ${ }^{8,11-15} \mathrm{Na}$ mesma linha está também um Relatório da Inspeção-Geral das Atividades em Saúde (IGAS) de 2010, que identificou a Ginecologia e Obstetrícia como a primeira especialidade afetada por processos judiciais em Portugal (num total de
30), seguida da Cirurgia Geral e da Ortopedia. ${ }^{4}$

Os queixosos mais frequentes foram doentes em nome próprio, seguidos de instituições. Este facto parece corroborar que as queixas que são apresentadas ao CD surgem principalmente no contexto da relação médico-doente e só depois no âmbito da relação com entidades públicas ou privadas. Ainda quanto aos queixosos, apenas uma minoria $(9,3 \%)$ recorreu a um advogado, o que se ficará a dever não só ao âmbito de ação do CD (nesta instância, não há normalmente necessidade de contratação de um advogado), mas também à realidade da prestação de serviços médicos em Portugal, que é muito distante de algumas sociedades mais litigantes, como os Estados Unidos da América. ${ }^{20}$ 
Quanto aos arguidos mais frequentes, o predomínio do género feminino e do escalão etário dos 41 aos 60 anos não nos parecem ser significativos, na medida em que a distribuição de género e idade, entre especialistas de Ginecologia e Obstetrícia, em 2011 , foi de $63 \%$ do sexo feminino versus $37 \%$ do masculino, com idades maioritariamente situadas no escalão etário referido. ${ }^{27,28}$ Khaliq et al relataram um aumento progressivo da taxa de ações disciplinares a cada intervalo de 10 anos após o término do curso, ${ }^{11}$ mas como foi referido, essa situação não parece ter tido significado na nossa amostra. De notar também, que oito queixas visavam internos de formação específica. De facto qualquer médico, incluindo internos, poderá ser responsabilizado disciplinarmente, sendo de notar que, de acordo com Domingues e col., se regista uma falta de perceção para a responsabilidade disciplinar junto dos internos da especialidade de Ginecologia e Obstetrícia. ${ }^{8}$

Os motivos clínicos mais frequentemente envolvidos nas queixas relacionaram-se principalmente com asfixia peri-natal, diagnóstico pré-natal, complicações materno-fetais de parto e complicações de cirurgias ginecológicas. As situações identificadas como motivo são consistentes com a bibliografia existente sobre queixas em Ginecologia e Obstetrícia. ${ }^{29-33}$

Das 54 queixas analisadas, 52 resultaram na não penalização do médico e foram arquivadas, enquanto que duas (uma por conflito médico/doente e outra por conluio médico/comércio) continuam a aguardar decisão. Assim, e apesar da Ginecologia e Obstetrícia ser uma especialidade propensa a queixas, o despacho de condenação emitido pelo CD da SRN da OM não reconheceu em nenhum caso matéria para punir disciplinarmente os médicos visados, o que está de acordo com alguma da bibliografia existente que reporta que, apesar do número de queixas ser elevado, as que motivam penalização do arguido e indemnização aos queixosos são em número bastante menor. ${ }^{12-15,24}$ Este aspeto deve ser visto com todo o cuidado, no que respeita às queixas relacionadas com alegada negligência médica. Estes casos são morosos e complexos, requerem normalmente a consulta de peritos dos Colégios de Especialidade e envolvem frequentemente tribunais. Nesse sentido, apesar de no período estudado os processos de alegada negligência terem sido todos arquivados pelo $C D$, isso não quer dizer que os médicos visados não tenham tido graves problemas com as situações suscitadas. Na verdade, tiveram frequentemente que recorrer a processos de defesa desgastantes, ${ }^{2}$ tendo sido expostos não só ao CD mas também a outras instâncias disciplinares e judiciais. ${ }^{1,18}$ Conforme discutido anteriormente, o arquivamento numa das instâncias não significa necessariamente o mesmo noutras. Outro aspeto a considerar é o facto de a Ginecologia e Obstetrícia lidar com situações de extrema sensibilidade pessoal e social, ${ }^{34}$ como as que se relacionam com as sequelas do parto, em que as limitações da medicina podem ser mal-entendidas pela comunidade, conduzindo nalguns casos a queixas em que se confundem os maus desfechos com negligência - queixas essas que, depois de analisadas, não configuram má prática. Na verdade, e especialmente em medicina materno-fetal, os maus desfechos nem sempre são evitáveis, mesmo quando se disponibilizam os melhores meios de atendimento possíveis.

\section{CONCLUSÕES}

A Ginecologia e Obstetrícia foi a quarta especialidade com maior risco de ação disciplinar na região norte de Portugal nos anos 2008 a 2012. Acresce que se considerarmos exclusivamente as queixas de alegada negligência, a Ginecologia e Obstetrícia foi a especialidade mais afetada, apesar de todos os processos, com exceção de dois ainda não concluídos, terem sido arquivados sem condenação do médico visado.

\section{AGRADECIMENTOS}

Ao Conselho Disciplinar da Seção Regional Norte da Ordem dos Médicos e em especial a António Mendes e Susana Costa pela colaboração prestada sem a qual este trabalho não teria sido possível. AAntónio Pinheiro pela ajuda na caracterização demográfica dos médicos da região norte de Portugal.

\section{CONFLITOS DE INTERESSE}

Os autores declaram a inexistência de conflitos de interesse.

\section{FONTES DE FINANCIAMENTO}

Não existiram fontes externas de financiamento para a realização deste artigo.

\section{REFERÊNCIAS}

1. Calvão FU. A responsabilidade médica e o direito administrativo. Rev Port Anest. 1999;115-32.

2. Seabury SA, Chandra A, Lakdawalla $D N$, Jena $A B$. On average, physicians spend nearly 11 percent of their 40-year careers with an open, unresolved malpractice claim. Health Aff. 2013;32:111-9.

3. Cabete J, Lencastre A, Apetato M, Paiva Lopes MJ. Why are our patients claiming? Statistics from a dermatology clinic. Acta Med Port. 2012;25;265-70.

4. Inspecção sobre monitorização do erro médico e acções judiciais com pedido de indemnização por deficiente assistência médica (follow-up). Processo n 44/2010. Lisboa: Inspecção-Geral das Actividades em Saúde; 2010.

5. Alam A, Khan J, Liu J, Klemensberg J, Griesman J, Bell CM.

Characteristics and rates of disciplinary findings amongst anesthesiologists by professional colleges in Canada. Can J Anaesth. 2013;60;1013-9.

6. Alam A, Kurdyak P, Klemensberg J, Griesman J, Bell CM. The characteristics of psychiatrists disciplined by professional colleges in Canada. PLoS One. 2012;7:e50558.

7. Alam A, Klemensberg J, Griesman J, Bell CM. The characteristics of physicians disciplined by professional colleges in Canada. Open Med. 2011;5:e166-72.

8. Domingues AP, Moura P, Vieira DN. Responsabilidade médica em Ginecologia/ Obstetrícia - perspectiva portuguesa. Acta Obstet Ginecol Port. 2009;3:24-34.

9. Estatuto Disciplinar dos Trabalhadores Que Exercem Funções 
Públicas - Lei n. ${ }^{\circ}$ 58/2008. Diário da República, $1^{\text {a }}$ série - n. ${ }^{\circ} 174 / 2008$.

10. Estatuto da Ordem dos Médicos, Decreto-lei 282/1977. Ministério dos Assuntos Sociais. Diário da República, $1^{\circ}$ série - n. ${ }^{0153 / 1977 . ~}$

11. Khaliq AA, Dimassi H, Huang CY, Narine L, Smego RA Jr. Disciplinary action against physicians: who is likely to get disciplined? Am J Med. 2005;118:773-7.

12. Gómez-Durán EL, Mulà-Rosías JA, Lailla-Vicens JM, Benet-Travé J, Arimany-Manso J. Analysis of obstetrics and gynecology professional liability claims in Catalonia, Spain (1986-2010). J Forensic Leg Med. 2013;20:442-6.

13. Jena $A B$, Seabury $S$, Lakdawalla $D$, Chandra $A$. Malpractice risk according to physician specialty. N Eng J Med. 2011;365:629-36.

14. Jena AB, Chandra A, Lakdawalla D, Seabury S. Outcomes of medical malpractice litigation against US physicians. Arch Intern Med. 2012;172:892-4.

15. Seabury $S$, Chandra A, Lakdawalla $D$, Jena $A B$. Defense costs of medical malpractice claims. N Engl J Med. 2012;366:1354-6.

16. Código Deontológico, Regulamento n. ${ }^{\circ} 14 / 2009$. Ordem dos Médicos. Diário da República. $2^{\mathrm{a}}$ série - $\mathrm{n} .^{\circ} 8 / 2009$.

17. Estatuto Disciplinar dos Medicos, Decreto-lei 217/1994. Ministério da Saúde. Diário da República, $1^{\text {a }}$ série - n. ${ }^{0} 192 / 1994$

18. Udelsmann A. Responsabilidade civil, penal e etica dos médicos. Rev Assoc Med Bras. 2002;48:172-82.

19. Boyaciyan K, Camano L. O perfil dos médicos denunciados que exercem ginecologia e obstetrícia no estado de São Paulo. Rev Assoc Med Bras. 2006;52:144-7.

20. Bal BS. An introduction to medical malpractice in the United States. Clin Orthop Relat Res. 2009;467:339-47.

21. Cuperus-Bosma JM, Hout FA, Hubben JH, van der Wal G. Views of physicians, disciplinary board members and practicing lawyers on the new statutory disciplinary system for health care in The Netherlands. Health policy. 2006;77:202-11.
22. Alhafaji FY, Frederiks BJ, Legemaate J. The Dutch system of handling complaints in health care. Med Law. 2009;28:241-55.

23. Sohn $\mathrm{DH}$. Negligence, genuine error, and litigation. Int J Gen Med. 2013;6:49-56

24. Studdert DM, Mello MM, Gawande AA, Gandhi TK, Kachalia A, Yoon $C$, et al. Claims, errors, and compensation payments in medical malpractice litigation. N Eng J Med. 2006;354:2024-33.

25. Fox R, Yelland A, Draycott T. Analysis of legal claims--informing litigation systems and quality improvement. BJOG. 2014;121:6-10.

26. Papadakis MA, Teherani A, Banach MA, Knettler TR, Rattner SL, Stern DT, et al. Disciplinary action by medical boards and prior behavior in medical school. N Eng J Med. 2005;353:2673-82.

27. Santana P, Peixoto H, Loureiro A, Costa C, Nunes C, Duarte N. Estudo de evolução prospectiva de médicos no Sistema Nacional de Saúde. Coimbra: Universidade de Coimbra para a Ordem dos Médicos. 2013.

28. Censos 2011 - resultados definitivos. Lisboa: INE; 2012

29. Edozien LC. Medico-legal issues in gynaecology. Obstet Gynaecol Reprod Med. 2012;22:273-8.

30. White AA, Pichert JW, Bledsoe SH, Irwin C, Entman SS. Cause and effect analysis of closed claims in obstetrics and gynecology. Obstet Gynecol. 2005;105:1031-8

31. Andreasen S, Backe B, Jørstad RG, Oian P. A nationwide descriptive study of obstetric claims for compensation in Norway. Acta Obstet Gynecol Scand. 2012;91:1191-5.

32. Andreasen S, Backe B, Oian P. Claims for compensation after alleged birth asphyxia: a nationwide study covering 15 years. Acta Obstet Gynecol Scand. 2014;93:152-8.

33. Skidmore FD. Medicolegal implications of laparoscopic technique. Ann R Coll Surg Engl. 2010;92:271.

34. Chou MM. Litigation in obstetrics: a lesson learnt and a lesson to share. Taiwanese J Obstet Gynecol. 2006;45:1-9. 
Francisco SILVA, Manuel RODRIGUES E RODRIGUES, João BERNARDES

\section{Ações Disciplinares em Ginecologia e Obstetrícia na Região Norte de Portugal nos Anos 2008 a 2012}

Acta Med Port 2015:28:194-203

Publicado pela Acta Médica Portuguesa, a Revista Científica da Ordem dos Médicos

Av. Almirante Gago Coutinho, 151

1749-084 Lisboa, Portugal.

Tel: +351218428 215

E-mail: submissao@actamedicaportuguesa.com

www.actamedicaportuguesa.com

ISSN:0870-399X | e-ISSN: 1646-0758

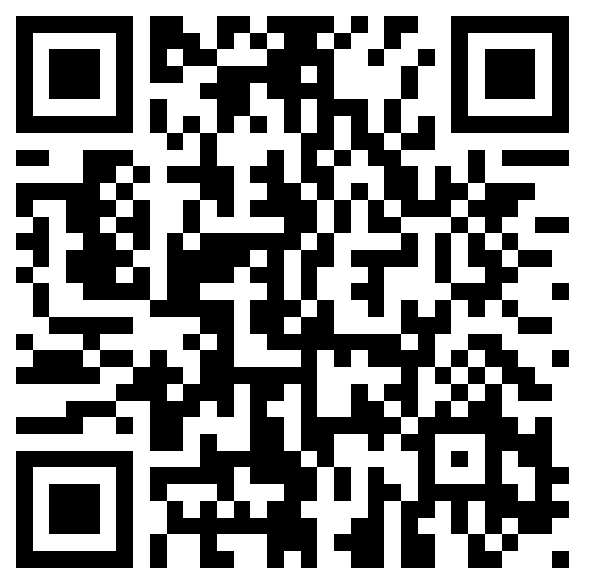

ACTA MÉDICA

PORTUGUESA 